\title{
Determination of HMG-CoA reductase inhibitors by micellar electrokinetic chromatography
}

\author{
Mircia Eleonora, Hancu Gabriel*, Rusu Aura, Cazacu Adriana, Balaci Teodora, Soare Ruxandra \\ University of Medicine and Pharmacy Tirgu Mures, Romania
}

\begin{abstract}
Objective: In this study we report the development of a simple, rapid and efficient capillary electrophoresis method for the simultaneous determination of atorvastatin, fluvastatin, lovastatin and simvastin.

Methods: Capillary zone electrophoresis proved to be efficient for the simultaneous separation of atorvastatin and fluvastatin, but could not resolve the determination of lovastatin and simvastatin. The simultaneous separation of all four statins was achieved by applying a micellar electrokinetic chromatographic method, after transforming lovastatin and simvastatin in $\beta$-hydroxyl acid forms through alkaline hydrolysis. The optimum electrophoretic conditions and analytical parameters were investigated and the analytical performances of the method were verified with regard to linearity, precision, accuracy, LOD and LOQ.

Results: The optimum electrophoretic separation conditions were: $25 \mathrm{mM}$ sodium tetraborate with $25 \mathrm{mM}$ sodium dodecyl sulphate buffer electrolyte at pH 9.5, applied voltage $+25 \mathrm{kV}$, separation temperature $25^{\circ} \mathrm{C}$, injection pressure/time $50 \mathrm{mbar} / 1$ minutes, UV detection at 230 $\mathrm{nm}$. Using the optimized electrophoretic conditions we succeeded in the simultaneous determination of the four statins in approximately 3 minutes, the order of migration being: atorvastatin, fluvastatin, lovastatin, simvastatin. The proposed method has been applied to the determination of the analytes in pharmaceutical tablets formulations.

Conclusions: The capillary electrophoretic method developed in the present work proved to be suitable for the routine analysis of statins and can be adopted as quality control protocol in pharmaceutical analysis.
\end{abstract}

Keywords: capillary electrophoresis, statins, micellar electrokinetic capillary chromatography, simultaneous determination, pharmaceutical analysis

Received: 11 November 2015 / Accepted: 19 December 2015

\section{Introduction}

Statins (3-Hydroxy-3-methylglutaryl (HMG) coenzyme A (COA) reductase inhibitors) are a class of hypolipidemic agents used in modern therapy to lower cholesterol levels by inhibiting HMG-CoA reductase, enzyme responsible for the conversion of HMG-CoA to mevalonate in the synthetic pathway of the cholesterol synthesis [1].

High cholesterol levels have been correlated with an increase in cardiovascular disease, statins being used for preventing and treating atherosclerosis that causes angina, heart attacks or strokes. Clinical trials in patients with and without coronary heart disease and with and without high cholesterol have demonstrated consistently that statins reduce the relative risk of major coronary events by $30 \%$ $[2,3]$.

Statins can be grouped into fermentation-derived and chemically synthetized derivatives, as lovastatin, pravastatin and simvastatin are derived from fungal fermentation, while atorvastatin, fluvastatin and rosuvastatin are entirely synthetic. Although all statins share a common mechanism of action and structural component that is very similar to the HMG portion of HMG-CoA reductase, they differ considerably in terms of their chemical structures. Depending upon their structural characteristics, statins exhibit different affinities towards HMG-CoA reductase and pharmacokinetic properties [4].

* Correspondence to: Gabriel Hancu

E-mail: gabriel.hancu@umftgm.ro
Lovastatin (LOVA) was the first commercially available compound for treatment of hypercholesterolemia, while simvastatin (SIMV) was obtained from LOV by replacement of 2-methylbutyryl side chain with 2,2-dimethylbutyryl group; both compounds are administered as lactone prodrugs and subsequently transformed to active metabolites [1].

Atorvastatin (ATOR) and fluvastatin (FLUV) are two frequently used highly effective synthetic HMG-CoA reductase inhibitors formulated in the pharmacological active open ring $\beta$-acid form; both derivatives possessing a heptanoic acid side chain with two hydroxyl groups at the $\mathrm{b}$ and $\delta$ positions, which is recognized by HMG-CoA reductase [1]. (Figure 1)

Various analytical methods for the determination of statins have been published before, the large majority being based on high-performance liquid chromatography (HPLC) [5,6]. However, capillary electrophoresis (CE) has become a useful tool in pharmaceutical analysis because of its advantages related to the high resolution, high selectivity, simplicity, short analysis time, cost efficiency and low consumption of solvents and reagents [7].

CE has been applied for the analysis of FLUV in a pharmaceutical capsule formulation and serum [8] and also for the chiral separation of FLUV enantiomers [9]. A micellar electrokinetic capillary chromatographic (MEKC) method has been applied for the simultaneous determination of ATOR and related substances [10]. In another study a CE method for the quantitation of ATOR in tablets was de- 
veloped and further miniaturized to a microchip platform with linear imaging UV detection using microchip electrophoresis [11]. A capillary zone electrophoresis (CZE) method was developed for the separation and simultaneous determination of ATOR and amlodipine from their combined fixed dose formulations [12]. A MEKC method was developed for the quantification of LOVA and SIMV in pharmaceutical dosage forms [13]. More recently a MEKC method for the determination of six different statins has been developed and applied for the analysis of the studied drugs in pharmaceutical samples [14].

Taking in consideration all the aspects presented the development of new methods of analysis of statins represents a necessity and also a challenge for the analysts. The purpose of this study was the development of a simple, rapid and efficient CE method for the simultaneous determination of ATOR, FLUV, LOVA and SIMV in a single run.

\section{Materials and Methods}

\section{Apparatus}

Experiments were performed with an Agilent (Waldbronn, Germany) 1600 CE system equipped with a diode-array detector. Separations were performed in $38.5 \mathrm{~cm}$ (effective length $30 \mathrm{~cm}) \times 50 \mu \mathrm{m}$ I.D. uncoated fused-silica capillaries (Agilent). Electropherograms were recorded and processed by use of Chemstation 7.01 software (Agilent). Buffer solution $\mathrm{pH}$ was determined with a Terminal 740 pH-meter (Inolab, Germany).

\section{Chemicals and Reagents}

The analyzed statins were purchased from different distributors: atorvastatin calcium (Morepen Laboratories, New Delhi, India), fluvastatin sodium (Neo-Dankong Pharmaceutical, Zhejiang, China), lovastatin (Recordati Indus-
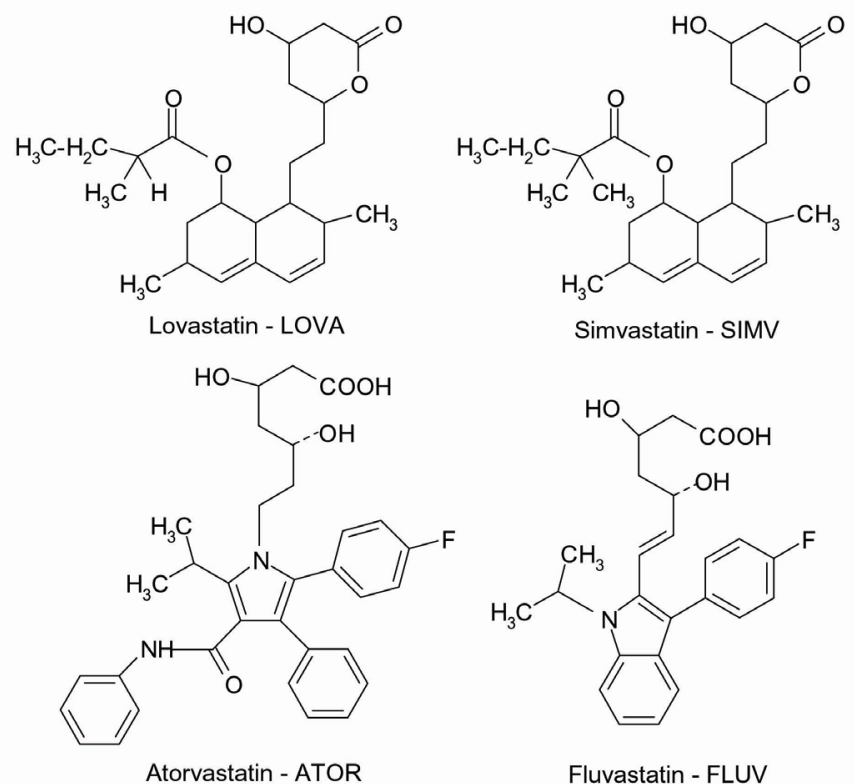

Fluvastatin - FLUV tria Chimica e Farmaceutica, Italia), simvastatin (Gedeon Richter, Tîrgu Mureș, Romania). The analytes were of pharmaceutical grade commplying with the stipulations of European Pharmacopoeia.

All reagents were of analytical grade quality: phosphoric acid (Chimopar, Bucharest, Romania), methanol, sodium hydroxide (Lach Ner, Neratovice, Czech Republic), sodium tetraborate, disodium hydrogenophosphate, sodium didydrogenophosphate, sodium dodecyl sulfate - SDS (Merck, Darmstadt Germany). Purified water was provided by a Milli-Q Plus water purification system (Millipore, Milford, MA, USA).

\section{Sample preparation}

Sample stock solutions were prepared by dissolving the analytes in methanol in a concentration of $100 \mathrm{mg} \mathrm{mL}^{-1}$ and later diluted with the same solvent to the appropriate concentration. All samples and buffers were filtered through a $0.45 \mu \mathrm{m}$ syringe filter and sonicated for 5 minutes before use. The samples were introduced in the system at the anodic end of the capillary by hydrodynamic injection.

\section{Electrophoretic conditions}

Before first use the capillary was flushed for 30 minutes with $0.1 \mathrm{M}$ sodium hydroxide followed by a 30 minutes rinsing with water and a 15 minute conditioning with the background electrolyte (BGE) used in the analysis. Between runs the capillary was preconditioned for 5 minute with the BGE, in order to maintain proper reproducibility of run-to-run injections.

In the preliminary analysis we applied some "standard" electrophoretic conditions for a CZE analysis: temperature $25^{\circ} \mathrm{C}$, applied voltage $+20 \mathrm{kV}$, injection pressure $\mathrm{x}$ time 50 mbar x 1 sec, sample concentration $25 \mu \mathrm{g} \mathrm{mL}^{-1}$.

Previously we recorded the UV spectra of the studied statins in methanol and found absorption maxima, at 247 $\mathrm{nm}$ for ATOR, 237 and $307 \mathrm{~nm}$ for FLUV, $242 \mathrm{~nm}$ for LOVA and $239 \mathrm{~nm}$ for SIMV; $240 \mathrm{~nm}$ was elected as the optimum wavelength for analysis.

\section{Results and Discussion}

\section{Preliminary Analysis}

In order to establish the electrophoretic behavior of the analytes and to find suitable electrophoretic conditions for the separation, preliminary experiments were conducted using background electrolytes (BGE) with different compositions at different $\mathrm{pH}$ values. In the preliminary analysis we used: $25 \mathrm{mM}$ phosphoric acid ( $\mathrm{pH} 2.1), 25 \mathrm{mM}$ sodium dihydrogen phosphate ( $\mathrm{pH}$ 5.0), $25 \mathrm{mM}$ disodium hydrogen phosphate-sodium dihydrogen phosphate $(1: 1)$ $(\mathrm{pH}$ 7.0) and $25 \mathrm{mM}$ sodium tetraborate $(\mathrm{pH}$ 9.3) as BGE; buffer $\mathrm{pH}$ was modified by addition of $0.1 \mathrm{M}$ sodium hydroxide solution.

The first step in optimizing the separation was the selection of an appropriate buffer electrolyte with an optimum

Fig. 1. Chemical structures of studied statins 
$\mathrm{pH}$, which determines the degree of ionization and consequently the electrohoretic mobility of the analytes. As reported in the literature ATOR has a pKa value of 4.33, FLUV 4.56, LOVA 4.18 while SIMV $4.21[15,16]$. ATOR and FLUV have in their structure a carboxylic group; consequently will be completely ionized in an anionic form when using an alkaline BGE ( $25 \mathrm{mM}$ sodium tetraborate, $\mathrm{pH}$ 9.3). LOVA and SIMV are in neutral lactone form, and exhibit no electrophoretic mobility migrating with together with the electroosmotic flow (EOF). In order to transform this neutral lactone forms in the corresponding ionizable acid forms alkaline hydrolisis was used, by adding $0.1 \mathrm{NaOH}$ solution to the LOVA and SIMV stock solutions (1:1). (Figure 2)

Open-ring hydroxyl acid forms of LOVA and SIMV are also negatively charged when using alkaline BGE and can be separated from other statins by CZE. However LOVA and SIMV couldn't be separated from each other in a mixture when using CZE, exhibiting the same electrophoretic mobility, due to very similar structural characteristics.

Migration times of the analytes decreased with an increase of the $\mathrm{pH}$ of the BGE, due to an increase of the apparent electrophoretic mobility of the analytes and generation of a strong EOF. The addition of an organic solvent (methanol) to the buffer electrolyte increased migration times of the analytes without resolving the separation between LOVA and SIMV.

In conclusion CZE can be used for the separation of ATOR and FLUV with a short analysis time and high resolution, but not for the simultaneous separation of LOVA and SIMV. (Figure 3)

In order to separate LOVA and SIMV, we applied a MEKC method, by adding an anionic surfactant (sodium dodecyl sulphate) to the buffer electrolyte. A change in the migration order between ATOR and FLUV was observed when applying MEKC conditions. The separation of LOVA and SIMV was resolved when using MEKC but only if was preceded by an alkaline hydrolysis; as the neutral lactone forms could not be separated. The best separation was achieved when using a buffer containing $25 \mathrm{mM}$

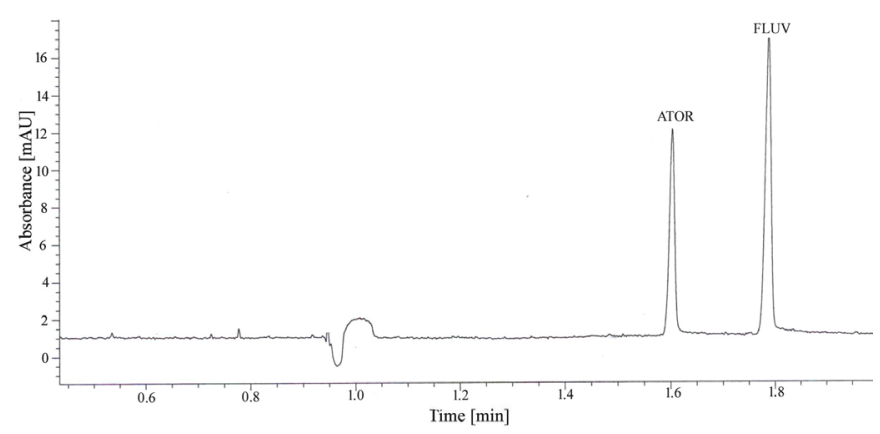

Fig. 2. Electropherogram of CZE separation of ATOR and FLUV. Electrophoretic conditions: $25 \mathrm{mM}$ sodium tetraborate buffer, $\mathrm{pH}$ 9.3 , voltage $+25 \mathrm{kV}$, temperature $25^{\circ} \mathrm{C}$, hydrodynamic injection 50 mbar/ 1 second, detection wavelength $240 \mathrm{~nm}$, statins concentration $25 \mu \mathrm{gL} \mathrm{m}^{-1}$ sodium tetraborate and $25 \mathrm{mM}$ sodium dodecyl sulphate at $\mathrm{pH} 9.3$.

\section{Optimization of the Analytical Conditions}

Selectivity in $\mathrm{CE}$ can be controlled by background electrolyte (BGE) concentration, $\mathrm{pH}$, buffer additives, applied voltage, temperature and injection parameters. All these parameters were varied and results are summarized below.

The effect of BGE concentration was studied over a concentration from 25 to $100 \mathrm{mM}$ at a constant $\mathrm{pH}$ of 9.3. Higher buffer concentration provided a lower EOF and resulted in more Joule heating which affected peak efficiency and migration times. Based on the migration times and generated current, a $25 \mathrm{mM}$ sodium tetraborate buffer was chosen.

The influence of SDS addition as surfactant in the running buffer was also studied. Increasing the SDS concentration in the BGE resulted in a slight improvement of the separation resolution but at the same time raised the current in the capillary and increased considerably the migration times. Therefore a concentration of $25 \mathrm{mM}$ SDS was chosen as a compromise between resolution and speed of analysis.

The addition of an organic modifier in the presence of SDS in the electrolyte solution increased migration times and also separation resolution but in the same time peak broadening was observed.

In order to determine to optimal applied voltage, the influence of voltage $(10-30 \mathrm{kV})$ on the migration time was investigated using the optimized BGE conditions. Increasing the voltage resulted in shorter migration times; thus a $+25 \mathrm{kV}$ voltage was selected as the optimum.

The influence of capillary temperature $\left(15-25^{\circ} \mathrm{C}\right)$ was evaluated under the chosen BGE conditions. When temperature was increased, migration times decreased; therefore a temperature of $25^{\circ} \mathrm{C}$ was chosen as the working temperature for the analysis.

For the determination of the optimal injection parameters, the influence of injection time (1-5 seconds) and injection pressure (30-50 mbar) was studied in order to attain low detection limits without affecting the quality of
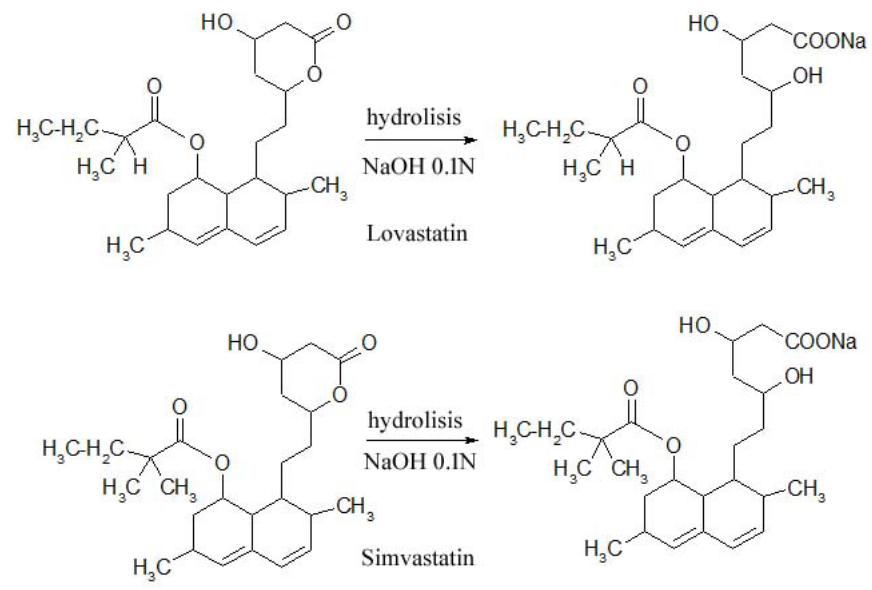

Fig. 3. Alkaline hydrolisis of LOVA and SIMV 


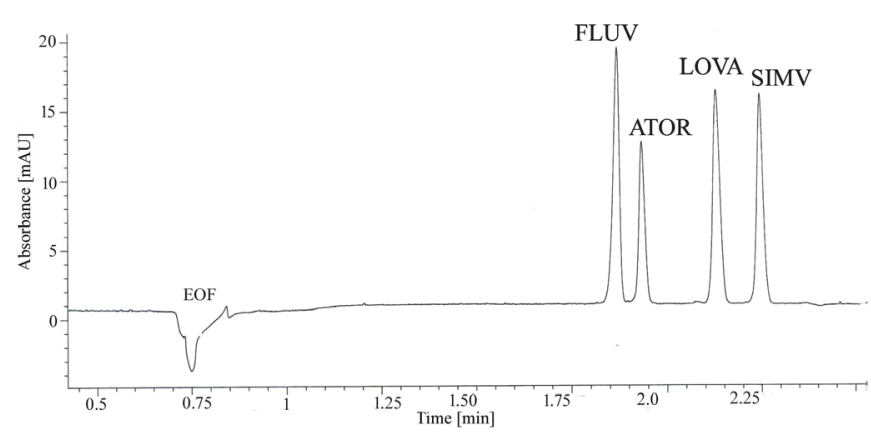

Fig. 4. Electropherogram of MEKC separation of the studiesd statins. Electrophoretic conditions: $25 \mathrm{mM}$ sodium tetraborate, $25 \mathrm{mM}$ sodium dodecyl sulphate buffer, $\mathrm{pH} 9.3$, voltage $+25 \mathrm{kV}$, temperature $25^{\circ} \mathrm{C}$, hydrodynamic injection $30 \mathrm{mbar} / 2$ seconds, detection wavelength $240 \mathrm{~nm}$, statins concentration $25 \mu \mathrm{g} \mathrm{mL}^{-1}$

the peak shapes and reproducibility, migration times, as well as resolution; a injection pressure of $30 \mathrm{mbar}$ for 2 seconds was selected as optimum.

The electropherogram obtained in the simultaneous separation of the four studied statins using the optimized analytical conditions is presented in figure 4 .

\section{Analytical Performance}

After method development, experiments were carried out to evaluate the validity of the method for the simultaneous determination of statins under optimized conditions.

The repeatability and intermediate precision for migration times and peak areas was determined by performing six runs of the sample $(\mathrm{n}=6, \mathrm{c}=25 \mu \mathrm{g} \mathrm{mL}-1)$ under the optimized conditions over two consecutive days (Table I).

The linearity of the method was investigated over a specific the concentration range $5-100 \mu \mathrm{g} \mathrm{mL}^{-1}$ on the basis of six measurement points and three triplicate analyses ( $\mathrm{n}$ = 3) per concentration (Table I).

LOD and LOQ were estimated as (standard deviation of regression equation)/(slope of the regression equation) $\times$ 3.3 and 10 , respectively (Table II).

Table I. Intra-day and inter-day precision of the migration times and peak areas for proposed method ( $c=25 \mu \mathrm{gL}^{-1}$ ).

\begin{tabular}{lcccccc}
\hline & \multicolumn{3}{c}{ Intra-day precision $(\mathrm{n}=6)$} & \multicolumn{3}{c}{ Inter-day precision $(\mathrm{n}=6)$} \\
\cline { 2 - 7 } Substance & $\begin{array}{c}\text { RSD (\%) } \\
\text { migra- } \\
\text { tion time }\end{array}$ & $\begin{array}{c}\text { RSD }(\%) \\
\text { peak } \\
\text { area }\end{array}$ & $\begin{array}{c}\text { RSD }(\%) \\
\text { peak } \\
\text { height }\end{array}$ & $\begin{array}{c}\text { RSD }(\%) \\
\text { migra- } \\
\text { tion time }\end{array}$ & $\begin{array}{c}\text { RSD }(\%) \\
\text { peak } \\
\text { area }\end{array}$ & $\begin{array}{c}\text { RSD (\%) } \\
\text { peak } \\
\text { height }\end{array}$ \\
\hline ATOR & 0.02 & 0.72 & 1.13 & 0.22 & 1.54 & 1.62 \\
FLUV & 0.02 & 0.62 & 1.46 & 0.25 & 1.48 & 1.78 \\
LOVA & 0.02 & 1.04 & 1.30 & 0.32 & 1.85 & 1.81 \\
SIMV & 0.02 & 1.06 & 1.39 & 0.40 & 1.92 & 1.88 \\
\hline
\end{tabular}

Table II. Regression and correlation data for the calibration curves of statins (concentration range $5-100 \mu \mathrm{g} \mathrm{mL}^{-1}, \mathrm{n}=6$ )

\begin{tabular}{lcccc}
\hline Substance & Regression equation & $\begin{array}{c}\text { Correlation } \\
\text { coefficient }\end{array}$ & $\begin{array}{c}\text { LOD } \\
(\mu \mathrm{g} \mathrm{mL}-1)\end{array}$ & $\begin{array}{c}\mathrm{LOQ} \\
(\mu \mathrm{g} \mathrm{mL}-1)\end{array}$ \\
\hline ATOR & $\mathrm{y}=0.253 \mathrm{x}+0.016$ & 0.997 & 3.58 & 10.86 \\
FLUV & $\mathrm{y}=0.442 \mathrm{x}-0.368$ & 0.997 & 4.09 & 12.41 \\
LOVA & $\mathrm{y}=0.417 \mathrm{x}-0.688$ & 0.996 & 5.44 & 16.50 \\
SIMV & $\mathrm{y}=0.384 \mathrm{x}-0.713$ & 0.995 & 5.62 & 17.03 \\
\hline
\end{tabular}

The robustness of the method was demonstrated by studying the influence of the variation of several experimental parameters like buffer concentration (20-30 mM), applied voltage $(25-30 \mathrm{kV})$ and temperature $\left(18-22^{\circ} \mathrm{C}\right)$, changing only one of these each time, and monitoring variation of the migration times. Only small acceptable deviations ( $\mathrm{RSD}<2 \%$ ) were observed indicating that the robustness of the method was good.

The accuracy of the method was determined by using recovery experiments analyzing solutions of known statin concentrations within the linearity range at three levels $\left(10,25\right.$ and $\left.50 \mu \mathrm{g} \mathrm{mL}^{-1}\right)$, the mean recovery was between $98.5-99.8 \%(\mathrm{n}=3$ at each concentration level), respectively, showing that the accuracy of the method was good.

\section{Conclusions}

Analytical methods for determination of statins are usually developed individually as expected from their different structural and chemical characteristics, and also because statins are not used in combination with other statin molecules during therapy. However, the development of a rapid analytical procedure that is not limited to the analysis of only one statin can be considered as a useful assessment in quality control.

A MEKC method for the simultaneous determination of ATOR, FLUV, LOVA and SIMV was successfully developed. Under the optimized conditions, baseline separation of the four analytes was obtained in approximately $3 \mathrm{~min}$ utes. Good analytical performance with regards of linearity, reproductibility, precision and accuracy was achieved. The good separation with high efficiency achieved in a very short analysis time represents the main advantage of the newly developed method.

The MEKC separation mechanism can be explained on the individual partitioning equilibrium of the analytes between the micellar and the aqueous phase; as the greater percentage of analyte is distributed into the micelle, the slower it will migrate. In our case the negatively charged statins were not strongly attracted to the anionic micelles, and consequently were separated as a result of differences in their electrophoretic mobilities and hidrophobicity.

The results of the study confirmed that the proposed method is well suited for the routine analysis of the studied analytes and therefore can be adopted as quality control protocol in pharmaceutical industry.

\section{Acknowledgments}

Our work was supported with a project funded through Internal Research Grants by the University of Medicine and Pharmacy of Tîrgu Mureş, Romania (grant contract for execution of research projects nr. 6/23.12.2014).

\section{Conflict of interest}

None to declare. 


\section{References}

1. Beale JH, Beale JM. Wilson and Gisvold's textbook of Organic Medicinal and Pharmaceutical Chemistry, 12th ed., Philadelphia, Lippincott Williams\&Wilkins; 2011.652-654.

2. Mc Taggart F, Jones P. Effects of statins on high-density lipoproteins: a potential contribution to cardiovascular benefit. Cardiovasc Drugs Ther. 2008;22:321-338

3. Balk EM, Karas $\mathrm{RH}$, Jordan $\mathrm{HS}$, et al. Effects of statins on vascular structure and function: a systematic review. Am J Med. 2004;117:775790.

4. Garcia I, Fall Y, Gomez G. Review of synthesis, biological assay, and QSAR studies of HMGR inhibitors. Curr Top Med Chem. 2012;12:895919.

5. Nirogi R, Mudigonda K, Kandikere V. Chromatography-mass spectrometry methods for the quantitation of statins in biological samples. J Pharm Biomed Anal. 2007;44:379-387.

6. Novakova L, Satinsly D, Solich P. HPLC methods for the determination of simvastatin and atorvastatin. Trend Anal Chem. 2008;27:352-366.

7. Deeb SE, Watzig H, El-Hady DA, et al. Recent advances in capillary electrophoretic migration techniques for pharmaceutical analysis. Electrophoresis. 2014;35:170-189.

8. Dogrokul-Ak D, Kircali K, Tuncel M, Aboul-Enein $\mathrm{H}$. Validated analysis of fluvastatin in a pharmaceutical capsule formulation and serum by capillary electrophoresis. Biomed Chromatogr. 2001;15:389-392.

9. Trung TQ, Dung PT, Hoan NN, et al. Chiral separation of fluvastatin enantiomers by capillary electrophoresis. Arch Pharm Res. 2008;31:1066-
1072.

10. Nigovic B, Damic M, Injac R, Glavac NK, Strukelj B. Analysis of atorvastatin and related substances by MEKC. Chromatographia. 2009;69:12991305.

11. Guinhen E, Sisk GD, Scully NM, Glennon JD. Rapid analysis of atorvastatin calcium using capillary electrophoresis and microchip electrophoresis. Electrophoresis. 2006;27:2338-2347.

12. Hefnawy MM, Sultan M, Al-Johar $H$. Development of capillary electrophoresis technique for simultaneous measurement of amlodipine and atorvastatin from their combination drug formulations. J Liq Chromatogr Relat Technol. 2009:32:2923-2942.

13. Srinivasu MK, Narasa Raju A, Om Reddy G. Determination of lovastatin and simvastatin in pharmaceutical dosage form by MEKC, J. Pharm. Biomed. Anal. 2002;29:715-721.

14. Damic M, Nigovic B. Fast analysis of statins in pharmaceuticals by MEKC. Chromatographia. 2010;71:233-240.

15. Miller JM, Blackburn AC, Shi Y, Melzak AJ, Ando HY. Semi-empirical relationships between effective mobility, charge, and molecular weight of pharmaceuticals by pressure-assisted capillary electrophoresis: applications in drug discovery. Electrophoresis. 2002;23:2833-2841.

16. Ishihama J, Nakamura M, Miwa T, Kajima T, Asakawa N. A rapid method for pKa determination of drugs using pressure-assisted capillary electrophoresis with photodiode array detection in drug discovery. J Pharm Sci. 2002;91:933-942. 